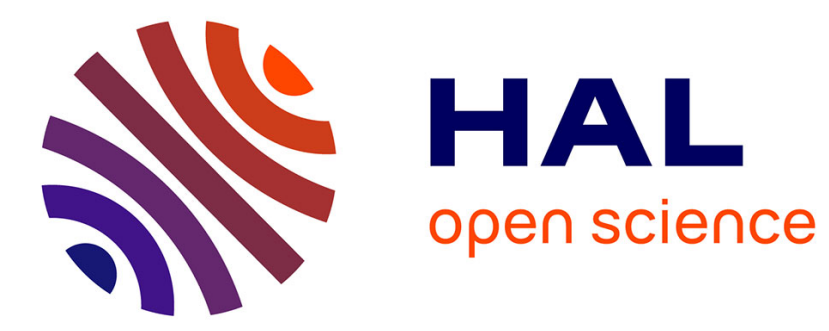

\title{
Nonlinear approximation with redundant dictionaries
} Lasse Borup, Morten Nielsen, Rémi Gribonval

\section{To cite this version:}

Lasse Borup, Morten Nielsen, Rémi Gribonval. Nonlinear approximation with redundant dictionaries. Acoustics, Speech and Signal Processing, 2005. ICASSP 2005. IEEE International Conference on, Mar 2005, Philadeplhia, PA, United States. pp.IV/261 - IV/264, 10.1109/ICASSP.2005.1415995 . inria-00564480

\section{HAL Id: inria-00564480 \\ https://hal.inria.fr/inria-00564480}

Submitted on 9 Feb 2011

HAL is a multi-disciplinary open access archive for the deposit and dissemination of scientific research documents, whether they are published or not. The documents may come from teaching and research institutions in France or abroad, or from public or private research centers.
L'archive ouverte pluridisciplinaire HAL, est destinée au dépôt et à la diffusion de documents scientifiques de niveau recherche, publiés ou non, émanant des établissements d'enseignement et de recherche français ou étrangers, des laboratoires publics ou privés. 


\section{NONLINEAR APPROXIMATION WITH REDUNDANT DICTIONARIES}

\author{
L. Borup, M. Nielsen \\ Aalborg University, Dept. of Math. \\ Fredrik Bajers Vej 7G \\ DK-9220 Aalborg East, Denmark \\ Email:\{lasse,mnielsen\}@math.aau.dk
}

\author{
R. Gribonval
}

\author{
IRISA \\ Campus de Beaulieu \\ F-35760 Rennes Cedex, France \\ Email: remi.gribonval@irisa.fr
}

\begin{abstract}
In this paper we study nonlinear approximation and data representation with redundant function dictionaries. In particular, approximation with redundant wavelet bi-frame systems is studied in detail. Several results for orthonormal wavelets are generalized to the redundant case. In general, for a wavelet bi-frame system the approximation properties are limited by the number of vanishing moments of the system. In some cases this can be overcome by oversampling, but at a price of replacing the canonical expansion by another linear expansion. Moreover, for special nonoversampled wavelet bi-frames we can obtain good approximation properties not restricted by the number of vanishing moments, but again without using the canonical expansion.
\end{abstract}

\section{INTRODUCTION}

Advances in communication, sensing, and computational power have led to an explosion of data. The size and varied formats for these datasets challenge existing techniques for transmission, storage, querying, display, and numerical manipulation. It is thus vital to develop efficient and robust data representations that lend themselves to scientific analysis and computation. An important theoretical tool along this line is the theory of constructive approximation.

Say we have an expansion of a target function $f$ in an ordered basis, and a positive integer $m$. By $m$-term nonlinear approximation we mean the process of approximating $f$ by truncating the expansion using only the $m$ "most important" terms. Important terms could be those minimizing a given cost function.

It is interesting to observe how the approximant changes as $m$ increases. In constructive approximation the target functions are divided into a family of approximation spaces classified by how fast the approximant converges to $f$ as $m$ increases. The study of these spaces is important for practical purposes since they offer some information on how

Research supported by the Danish Technical Science Foundation, Grant no. 9701481. much we can expect to be able to compress a given function without substantial loss of information.

For nice systems such as trigonometric- and wavelet bases, there already exist characterizations of the corresponding approximation spaces [1]. However, if these systems are replaced by more general dictionaries, the picture becomes less clear. Examples of structured redundant dictionaries are wavelet bi-frame systems and Gabor frames. The first system has the same basic structure as a wavelet system, while the latter consists of local trigonometric functions.

Many open problems remain regarding function representation using redundant dictionaries. But there is no doubt that sparse representations using such systems will have numerous applications, in fact, there is a growing interest within signal and image processing communities in this approach.

In this note we study the approximation properties of wavelet bi-frame systems in details. The results extends the known results for wavelets to the redundant case.

\section{APPROXIMATION SPACES}

In this section we introduce the theoretical tools to study nonlinear approximation with redundant function dictionaries. A dictionary $\mathcal{D}=\left\{g_{k}\right\}_{k \in \mathbb{N}}$ in $L_{p}$ is a countable collection of normalized elements from $L_{p}$. For $\mathcal{D}$ we consider the collection of all possible $m$-term expansions with elements from $\mathcal{D}$ :

$$
\Sigma_{m}(\mathcal{D}):=\left\{\sum_{i \in \Lambda} c_{i} g_{i} \mid c_{i} \in \mathbb{C}, \operatorname{card} \Lambda \leq m\right\} .
$$

The error of the best $m$-term approximation to an element $f \in L_{p}$ is then

$$
\sigma_{m}(f, \mathcal{D})_{p}:=\inf _{f_{m} \in \Sigma_{m}(\mathcal{D})}\left\|f-f_{m}\right\|_{L_{p}}
$$

The approximation space $\mathcal{A}_{q}^{\alpha}\left(L_{p}, \mathcal{D}\right)$ essentially consists of all signals that can be approximated at the rate $O\left(m^{-\alpha}\right)$. The parameter $q$ is for fine-tuning only. 
Definition 2.1 (Approximation spaces) The approximation space $\mathcal{A}_{q}^{\alpha}\left(L_{p}, \mathcal{D}\right)$ is defined by

$$
|f|_{\mathcal{A}_{q}^{\alpha}\left(L_{p}, \mathcal{D}\right)}^{q}:=\sum_{m \in \mathbb{N}}\left(m^{\alpha} \sigma_{m}(f, \mathcal{D})_{p}\right)^{q} \frac{1}{m}<\infty,
$$

and (quasi)normed by $\|f\|_{\mathcal{A}_{q}^{\alpha}\left(L_{p}, \mathcal{D}\right)}=\|f\|_{p}+|f|_{\mathcal{A}_{q}^{\alpha}\left(L_{p}, \mathcal{D}\right)}$, for $0<q, \alpha<\infty$. When $q=\infty$, the $\ell_{q}$ norm is replaced by the sup-norm.

In what follows, we use the notation $V \hookrightarrow W$ to indicate $V$ is continuously embedded in $W$ for two (quasi)normed spaces $V$ and $W$, i.e., $V \subset W$ and there is a constant $C<$ $\infty$ such that $\|\cdot\|_{W} \leq C\|\cdot\|_{V}$.

It is known that the main tool in the characterization of $\mathcal{A}_{q}^{\alpha}\left(L_{p}, \mathcal{D}\right)$ comes from the link between approximation theory and interpolation theory (see e.g. [2, Theorem 9.1, Chapter 7]). Let $Y_{p}$ be a (quasi)Banach space continuously embedded in $L_{p}$ with semi-(quasi)norm $|\cdot|_{Y_{p}}$. Given $\alpha>0$, the Jackson inequality

$$
\sigma_{m}(f, \mathcal{D})_{p} \leq C m^{-\alpha}|f|_{Y_{p}}, \quad \forall f \in Y_{p}, \forall m \in \mathbb{N}
$$

and the Bernstein inequality

$$
|S|_{Y_{p}} \leq C^{\prime} m^{\alpha}\|S\|_{p}, \quad \forall S \in \Sigma_{m}(\mathcal{D})
$$

(with some constants $C$ and $C^{\prime}$ independent of $f, S$ and $m$ ) imply, respectively, the continuous embedding

$$
\left(L_{p}, Y_{p}\right)_{\beta / \alpha, q} \hookrightarrow \mathcal{A}_{q}^{\beta}\left(L_{p}, \mathcal{D}\right)
$$

and the converse embedding

$$
\left(L_{p}, Y_{p}\right)_{\beta / \alpha, q} \hookleftarrow \mathcal{A}_{q}^{\beta}\left(L_{p}, \mathcal{D}\right)
$$

for all $0<\beta<\alpha$ and $q \in(0, \infty]$. Here $(X, Y)_{\theta, q}$ denotes the real interpolation space between the Banach spaces $X$ and $Y$. We refer the reader to [3] for the definition of the real method of interpolation.

\section{WAVELET BI-FRAME SYSTEMS}

A dictionary of particular interest is a wavelet bi-frame. The redundant representation offered by wavelet bi-frames has already been put to good use for signal denoising, and is currently explored for image compression. Motivated by these and other applications, we would like to investigate the approximation properties of wavelet bi-frames. In particular, we are interested in wavelet bi-frames based on a multiresolution analysis, since this guarantees the existence of fast implementation algorithms.

Given a finite collection of functions $\Psi=\left\{\psi^{\ell}\right\}_{\ell \in E}$, $E:=\{1,2, \ldots, L\}$, in $L_{2}$, we use the notation $X(\Psi)$ to denote the corresponding "wavelet" system,

$$
X(\Psi):=\left\{\psi_{j, k}^{\ell} \mid j, k \in \mathbb{Z}, \ell \in E\right\},
$$

where $\psi_{j, k}:=2^{j / 2} \psi\left(2^{j} \cdot-k\right)$. A wavelet bi-frame for $L_{2}$ consists of two sequences of wavelets $\Psi=\left\{\psi^{\ell}\right\}_{\ell \in E}$ and $\tilde{\Psi}=\left\{\tilde{\psi}^{\ell}\right\}_{\ell \in E}$ for which the systems $X(\Psi)$ and $X(\tilde{\Psi})$ are Bessel systems, and satisfy the reconstruction formula

$$
f=\sum_{\ell \in E} \sum_{j, k \in \mathbb{Z}}\left\langle f, \tilde{\psi}_{j, k}^{\ell}\right\rangle \psi_{j, k}^{\ell}, \quad \forall f \in L_{2} .
$$

This definition implies that both $X(\Psi)$ and $X(\tilde{\Psi})$ are frames for $L_{2}$ and in fact the roles of $\Psi$ and $\tilde{\Psi}$ are interchangeable in (3). The special case with $\Psi=\tilde{\Psi}$ corresponds to a socalled tight wavelet frame.

The most common method for constructing wavelet biframes relies on so-called extension principles. The resulting bi-frames are based on a multiresolution analysis (MRA), and the generators are called bi-framelets. When the wavelet bi-frame is based on an MRA there exist $\phi, \tilde{\phi} \in L_{2}$ and $2 \pi$ periodic functions $\tau_{\ell}, \tilde{\tau}_{\ell}, \ell=0,1, \ldots, L$, such that

$$
\begin{aligned}
& \hat{\phi}(2 \xi)=\tau_{0}(\xi) \hat{\phi}(\xi), \\
& \hat{\psi}^{\ell}(2 \xi)=\tau_{\ell}(\xi) \hat{\phi}(\xi), \quad \text { and } \quad \hat{\tilde{\psi}}^{\ell}(2 \xi)=\tilde{\tau}_{\ell}(\xi) \hat{\tilde{\phi}}(\xi) \text {. }
\end{aligned}
$$

The construction of multiresolution-based wavelet frames have been studied extensively, see e.g. [4, 5, 6].

\section{JACKSON AND BERNSTEIN INEQUALITIES FOR WAVELET BI-FRAMES}

In this section we consider Jackson and Bernstein inequalities for wavelet bi-frames. Denote by $W^{s}\left(L_{\infty}\right)$ the Sobolev space consisting of functions with all $s$ distributional derivatives in $L_{\infty}$.

Proposition 4.1 ([7]) Let $X(\Psi), X(\tilde{\Psi})$ be a bi-framelet system and assume that $X(\Psi)$ is based on a compactly supported refinable function $\phi \in W^{s}\left(L_{\infty}\right), s \geq 0$. Then the Bernstein inequality

$$
|S|_{B_{\tau}^{d \alpha}\left(L_{\tau}\right)} \leq C m^{\alpha}\|S\|_{L_{p}}, \quad \forall S \in \Sigma_{m}(X(\Psi)),
$$

holds true for each $m \geq 1,0<\alpha<s, 0<p \leq \infty$, with $1 / \tau:=\alpha+1 / p$ and $C=C(\alpha, p)$.

A function $f$ is said to have $N \in \mathbb{N}$ vanishing moments if $\int_{\mathbb{R}} x^{k} f(x) d x=0$ for $k=0,1, \ldots, N-1$.

The following Jackson inequality is valid.

Proposition 4.2 ([7]) Let $X(\Psi), X(\tilde{\Psi})$ be a bi-frame. Suppose each $\tilde{\psi} \in \tilde{\Psi}$ has $N \in \mathbb{N}$ vanishing moments and $|\tilde{\psi}(x)| \leq C(1+|x|)^{-2(N+1)}$, and suppose there exist $\beta, \varepsilon>$ 0 such that for all $\psi \in \Psi \cup \tilde{\Psi}, \psi \in C^{\beta}$ and $|\psi(x)| \leq$ $C(1+|x|)^{-1-\varepsilon}$. Then, we have the Jackson inequality

$$
\sigma_{m}(f, X(\Psi))_{p} \leq C m^{-\alpha}\|f\|_{B_{\tau}^{d \alpha}\left(L_{\tau}\right)}
$$

for $p \in(1, \infty), \alpha<N$, and $1 / \tau=\alpha+1 / p$. 
Remark 4.3 It was shown in [7] that the rate of approximation given by Proposition 4.2 can be obtained simply by thresholding the canonical frame expansion (for sufficiently "nice" bi-frame systems).

Finally, combining Proposition 4.1 and Proposition 4.2 above, we obtain a full characterization of the approximationspaces using wavelet bi-frames.

Theorem 4.4 Let $X(\Psi), X(\tilde{\Psi})$ be a wavelet bi-frame system that satisfies the hypothesis of Propositions 4.1 and 4.2. Let $p \in(1, \infty)$ and $\tau:=(\alpha+1 / p)^{-1}$ where we assume $0<$ $\alpha<\min \{s, N\}$. Then, for each $0<\beta<\alpha, q \in(0, \infty]$, we have the characterization

$$
\mathcal{A}_{q}^{\beta}\left(L_{p}, X(\Psi)\right)=\left(L_{p}, B_{\tau}^{\alpha}\left(L_{\tau}\right)\right)_{\beta / \alpha, q} .
$$

Remark 4.5 It is known (see, e.g., [1]) that the right hand side of (5) equals the Besov space $B_{q}^{\beta}\left(L_{q}\right)$ when $1 / q=$ $\beta+1 / p, 0<\beta<\alpha$, and $q \in(0, \infty]$.

\section{BEYOND THE CANONICAL FRAME DECOMPOSITION}

In the proof of Proposition 4.2 in [7] it is essential to require that the dual system $X(\tilde{\Psi})$ has a sufficient number of vanishing moments. The remainder of this paper is devoted to study ways of avoiding this requirement since many biframelet systems only have few vanishing moments. The key is to oversample the system. Given a finite collection of functions $\Psi=\left\{\psi^{\ell}\right\}_{\ell \in E}$ in $L_{2}$ and $R \geq 1$ we let $X_{R}(\Psi)$ denote the oversampled system,

$$
X_{R}(\Psi):=\left\{2^{j / 2} \psi^{\ell}\left(2^{j} \cdot-k / R\right): j, k \in \mathbb{Z}, \ell \in E\right\} .
$$

It turns out that if $X(\Psi)$ is a frame in $L_{2}$ so is the oversampled system $X_{R}(\Psi)$. Moreover, if $X(\Psi), X(\tilde{\Psi})$ is a "nice" bi-framelet system, the oversampled system $X_{2^{N}}(\Psi), N \in$ $\mathbb{N}$, again, gives rise to an approximation space no larger than a Besov space, i.e. the Bernstein inequality proven in Proposition 4.1 holds true for the oversampled system.

Again, in order to get a complete characterization of the approximation spaces based on $X_{R}(\Psi)$ in terms of Besov spaces, we need to prove a matching Jackson estimate. The following result was given in [7].

Proposition 5.1 Let $X(\Psi), X(\tilde{\Psi})$ be a wavelet bi-frame system, $X(\eta)$ a bi-orthogonal wavelet basis and $r>0$ such that the Besov space $B_{\tau}^{r}\left(L_{\tau}\right), 0<\tau<\infty$, can be characterized by

$$
\left\{f \in L_{\tau}: \sum_{j, k \in \mathbb{Z}}\left(2^{j(r+1 / 2-1 / \tau)}\left|\left\langle f, \eta_{j, k}\right\rangle\right|\right)^{\tau}<\infty\right\} .
$$

Assume there exists a sequence $\left\{d_{k}^{\ell}\right\}_{\ell \in E, k \in \mathbb{Z}} \in \ell_{1 /(r+1)}$, such that

$$
\eta(x)=\sum_{\ell \in E} \sum_{k \in \mathbb{Z}} d_{k}^{\ell} \psi^{\ell}(x-k / R) .
$$

Then, for $1<p<\infty$, and $0<\alpha=1 / \tau-1 / p<r$, we have the Jackson inequality

$$
\sigma_{m}\left(f, X_{R}(\Psi)\right)_{p} \leq C m^{-\alpha}\|f\|_{B_{\tau}^{\alpha}\left(L_{\tau}\right)} .
$$

Now, by combining Proposition 4.1 (for the oversampled system) and Proposition 5.1 we obtain a complete characterization of the approximation spaces $\mathcal{A}_{q}^{\beta}\left(L_{p}, X_{2}(\Psi)\right)$, not limited by the number of vanishing moments of the dual system $\tilde{\Psi}$.

In our strategy to get a Jackson inequality for the (oversampled) framelet system $X_{R}(\Psi)$, the crucial issue is to identify some "nice" wavelet(s) that can be expanded sparsely in terms of the oversampled bi-frame system. For splinebased tight framelets, it was shown in [8] how to get a finite expansion of a nice semi-orthogonal wavelet in the twice oversampled $(R=2)$ framelet system. In [7] a construction is given which is valid for more general bi-framelets and only requires $R=2$.

Proposition 5.2 Let $X(\Psi), X(\tilde{\Psi})$ be an MRA-based wavelet bi-frame system and let $\phi$ and $\eta$ be respectively the scaling function and the associated standard orthonormal wavelet. Suppose that each filter $\tau_{\ell}, \ell=0,1, \ldots, L$, is a trigonometric polynomial,

$$
\sum_{\ell \in E}\left|\tau_{\ell}(\xi)\right|^{2}>0, \quad \text { for } \xi \neq 0,
$$

and $\phi$ is an $r$-regular scaling function (not necessarily orthonormal). Then there exists $\left\{d_{k}^{\ell}\right\} \in \bigcap_{\tau>0} \ell_{\tau}$ such that

$$
\eta(x):=\sum_{\ell \in E} \sum_{k \in \mathbb{Z}} d_{k}^{\ell} \psi^{\ell}(x-k / 2) .
$$

\subsection{Wavelets build with no oversampling}

We conclude by improving the result from Proposition 5.2 by showing that in certain cases no oversampling is needed to build a wavelet out of the framelet system.

The following lemma is a restatement of the so-called oblique extension principle, see [5].

Lemma 5.3 Let $X(\Psi)$ be a tight framelet system with associated trigonometric polynomials $\tau_{\ell}, \ell=0,1, \ldots, L$. Define $m_{\ell}\left(e^{-i \xi}\right):=\tau_{\ell}(\xi)$. Then there exists a rational function $T(z)$, with $T(1)=1$, such that

$$
\begin{aligned}
& m_{0}(z) \overline{m_{0}\left(z^{-1}\right)} T\left(z^{2}\right)+\sum_{\ell \in E} m_{\ell}(z) \overline{m_{\ell}\left(z^{-1}\right)}=T(z), \\
& m_{0}(z) \overline{m_{0}\left(-z^{-1}\right)} T\left(z^{2}\right)+\sum_{\ell \in E} m_{\ell}(z) \overline{m_{\ell}\left(-z^{-1}\right)}=0 .
\end{aligned}
$$


From Lemma 5.3 we can deduce the following result. Let $G(z)=\operatorname{gcd}\left\{m_{\ell}(z): \ell \in E\right\}$.

Theorem 5.4 Suppose $m_{0}(z)=\left(\frac{1+z}{2}\right)^{r} R(z)$, for some $r \in \mathbb{N}$, where $|R(1)|=1$ and $R(-1) \neq 0$. Then $G(z)=$ $(1-z)^{n} \tilde{G}(z)$ for some $1 \leq n \leq r$, where $\tilde{G}(1) \neq 0$.

Proof. By (7) we have

$$
\sum_{\ell \in E}\left|m_{\ell}(1)\right|^{2}=1-\left|m_{0}(1)\right|^{2}=1-|R(1)|^{2}=0 .
$$

Thus $(1-z) \mid G(z)$, i.e., $n \geq 1$.

Now, suppose the lemma holds for some $n \geq 1$. Then (8) yields $(1-z)^{n}\left(1+z^{-1}\right)^{n}=(-1)^{n}(1+z)^{n}\left(1-z^{-1}\right)^{n}$ is a factor of $m_{0}(z) m_{0}\left(-z^{-1}\right) T\left(z^{2}\right)$. Since $T(1)=1$, $R(1) \neq 0$ and $R(-1) \neq 0$, this implies $n \leq r$.

Let $\phi$ be a scaling function generated by the refinement filter $\tau_{0}(\xi)$, and let $P(\xi):=\sum_{k \in \mathbb{Z}}|\hat{\phi}(\xi-k)|^{2}$. Define

$$
\tilde{\tau}(\xi):=\frac{e^{-i \xi} \overline{\tau_{0}(\xi+\pi)} \sqrt{P(\xi+\pi)}}{\sqrt{P(2 \xi) P(\xi)}},
$$

and $M\left(e^{-i \xi}\right)=\tilde{\tau}(\xi)$. Notice that if $m_{0}(z)=\left(\frac{1+z}{2}\right)^{r} R(z)$ for some $r \in \mathbb{N}$, then $(1-z)^{r}$ is a factor of $M(z)$.

Recall that any orthonormal wavelet $\eta$ associated with the scaling function $\phi$ is given by

$$
\hat{\eta}(2 \xi)=v(2 \xi) \tilde{\tau}(\xi) \hat{\phi}(\xi)
$$

for some $2 \pi$-periodic function $v$ with $|v|=1$ a.e..

Let us try to expand such a wavelet in terms of integer shifts of the framelets $\psi^{\ell}$. A neccessary and sufficient condition for such an expansion to be possible is the existence of polynomials $p^{\ell}(z)$ such that $\hat{\eta}(\xi)=\sum_{\ell \in E} p^{\ell}\left(e^{-i \xi}\right) \hat{\psi}^{\ell}(\xi)$. Using (9) this is equivalent to the existence of polynomials $p^{\ell}(z)$ such that

$$
\sum_{\ell \in E} p^{\ell}\left(e^{-i 2 \xi}\right) \tau^{\ell}(\xi)=v(2 \xi) \tilde{\tau}(\xi) .
$$

According to Theorem 5.4 we have $m_{\ell}(z)=(1-z)^{n}$. $\left(a^{\ell}\left(z^{2}\right)+z b^{\ell}\left(z^{2}\right)\right)$ for some $1 \leq n \leq r$. Likewise we can write $M(z)=(1-z)^{n}\left(A\left(z^{2}\right)+z B\left(z^{2}\right)\right)$. Thus, with $q\left(e^{-i \xi}\right):=v(\xi),(10)$ can be rewritten

$$
q(z)\left[\begin{array}{c}
A(z) \\
B(z)
\end{array}\right]=\left[\begin{array}{lll}
a^{1}(z) & \cdots & a^{L}(z) \\
b^{1}(z) & \cdots & b^{L}(z)
\end{array}\right]\left[\begin{array}{c}
p^{1}(z) \\
\vdots \\
p^{L}(z)
\end{array}\right] .
$$

Now, using Proposition 4.1 and Proposition 5.1 we obtain.

Theorem 5.5 Let $X(\Psi)$ be a tight framelet system based on a compactly supported refinable function $\phi \in W^{s}\left(L_{\infty}\right)$, $s \geq 0$. Suppose the wavelet $\eta$ given by (9) can be used to characterize the Besov space $B_{\tau}^{r}\left(L_{\tau}\right)$ as given in (6), and suppose there exist polynomials $\left\{p^{\ell}(z)\right\}_{\ell \in E}$ such that (11) is satisfied. Let $p \in(1, \infty), \alpha<\min \{s, r\}$, and $\tau:=$ $(\alpha+1 / p)^{-1}$. Then, for each $0<\beta<\alpha$ and $q \in(0, \infty]$, we have the characterization

$$
\mathcal{A}_{q}^{\beta}\left(L_{p}, X(\Psi)\right)=\left(L_{p}, B_{\tau}^{\alpha}\left(L_{\tau}\right)\right)_{\beta / \alpha, q} .
$$

\section{CONCLUSION}

We have studied nonlinear approximation with redundant wavelet bi-frame systems. Several results for orthonormal wavelets have been extended to the redundant case. In particular, the approximation spaces for such systems have been completely characterized. For "nice" wavelet bi-frames, the optimal approximation rate can be obtained simply by thresholding the canonical frame decomposition of a given signal.

For oversampled wavelet bi-frame systems, the approximation results are obtained without requireing vanishing moments. It is also shown that an oversampling ratio of two is sufficient. For special wavelet bi-frames, oversampling is not needed at all to obtain the approximation results.

\section{REFERENCES}

[1] Ronald A. DeVore, "Nonlinear approximation," in Acta numerica, 1998, pp. 51-150. Cambridge Univ. Press, Cambridge, 1998.

[2] Ronald A. DeVore and George G. Lorentz, Constructive approximation, Springer-Verlag, Berlin, 1993.

[3] Colin Bennett and Robert Sharpley, Interpolation of operators, Academic Press Inc., Boston, MA, 1988.

[4] Charles K. Chui, Wenjie He, and Joachim Stöckler, "Tight frames with maximum vanishing moments and minimum support," in Approximation theory, X (St. Louis, MO, 2001), Innov. Appl. Math., pp. 187-206. Vanderbilt Univ. Press, Nashville, TN, 2002.

[5] Ingrid Daubechies, Bin Han, Amos Ron, and Zuowei Shen, "Framelets: MRA-based constructions of wavelet frames," Appl. Comput. Harmon. Anal., vol. 14, no. 1, pp. 1-46, 2003.

[6] Amos Ron and Zuowei Shen, "Affine systems in $L_{2}\left(\mathbf{R}^{d}\right)$ : the analysis of the analysis operator," J. Funct. Anal., vol. 148, no. 2, pp. 408-447, 1997.

[7] Lasse Borup, Rémi Gribonval, and Morten Nielsen, "Bi-framelet systems with few vanishing moments characterize Besov spaces," Appl. Comput. Harmon. Anal., vol. 17, no. 1, pp. 3-28, 2004.

[8] Rémi Gribonval and Morten Nielsen, "On approximation with spline generated framelets," Constr. Approx., vol. 20, no. 2, pp. 207-232, 2004. 